\title{
Application of a hepatitis $E$ transmission model to assess intervention strategies in a displaced persons camp in Uganda
}

\author{
$\underline{\text { G.N. }}$ Mercer $^{\mathrm{a}}$, M.R. Siddiqui ${ }^{\mathrm{b}}$ \\ ${ }^{a}$ National Centre for Epidemiology and Population Health, Australian National University, Canberra, \\ ACT, 0200, Australia \\ ${ }^{\mathrm{b}}$ Médecins Sans Frontières, Saffron Hill, London, United Kingdom \\ Email: Geoff.Mercer@anu.edu.au
}

\begin{abstract}
Hepatitis E virus is an enterically transmitted disease that mainly effects people in developing countries. It is known to spread through exposure to contaminated water sources (Balayan, 1997) and is also thought to spread through person-to-person transmission (Teshale et al., 2010). In recent years there have been outbreaks in internally displaced persons (IDP) camps in Sudan (Boccia et al., 2006; Guthmann et al., 2006) and Uganda (Siddiqui, 2010). In IDP camps interventions such as installation of hand washing points, water purification and latrine building are undertaken to control the spread of the disease. Hand washing points reduce the person-to-person transmission, water purification reduces exposure to the contaminated water source and latrine building reduces shedding of the virus by infected individuals to the environmental reservoir. Of interest is how effective these interventions are, which intervention should take precedence and how rapidly the interventions should be implemented.
\end{abstract}

A differential equation based compartment model is developed for the transmission of hepatitis $\mathrm{E}$ via the faecal-oral route including both an environmental reservoir and person-to-person spread. Due to the long incubation and infective periods of hepatitis E, the model includes both exposed and infectious classes of individuals. Multiple exposed and infective classes are used to give more realistic gamma distributed infective periods.

Where possible, parameters in the model are determined from knowledge of the aetiology of hepatitis E. The remaining unknown parameters are estimated by fitting to incidence data from four IDP camps during the recent Ugandan outbreak. This is performed using a Bayesian Markov chain Monte Carlo method where prior distributions for the unknown parameters are progressively refined until a posterior estimate of the parameter distributions is obtained.

Consistent estimates for the parameter governing transmission from the environment to an individual are found for all four camps. Shedding to the environmental reservoir was more variable across the four camps. The person-to-person parameter showed the greatest variation across the camps. This is consistent with conflicting reports in the literature as to the importance of person-to-person transmission of hepatitis $\mathrm{E}$ and is clearly an area requiring further research.

Before the model can be run to determine the effectiveness of interventions the timing of these interventions must be determined. A threshold number of cases need to be reported before interventions are considered and then there are various lags before interventions can be fully implemented. These lags include delays in testing, hardware delivery and installation and manpower and resource limitations. These delays are specific to each site and need to be determined in consultation with the field epidemiologists.

Keywords: Hepatitis E, mathematical model, infectious disease, faecal-oral, environmental reservoir, Monte Carlo, intervention 


\section{MODEL DEVELOPMENT}

A differential equation based compartment model is developed for the transmission of a disease via both an environmental reservoir and person-to-person spread. Recently related models have been developed for a number of diseases spread via the faecal-oral route that include an environmental reservoir in their transmission pathway. These include for hepatitis A (Ajelli et al., 2008), cholera (Codeço, 2001; Hartley et al., 2006; King et al., 2008; Andrews and Basu, 2011) and avian influenza amongst water fowl (Rohani et al., 2009; Breban et al., 2009).

The simplest compartmental disease transmission model that includes an environmental reservoir needs four compartments. Three of these compartments relate to the disease status in an individual with Susceptible (S), Infective (I) and Recovered (R) classes. The additional compartment gives the viral load in the environmental reservoir $(\mathrm{V})$. The differential equations governing transmission in this simple model are

$$
\begin{aligned}
\frac{d S}{d t} & =-\frac{\beta S I}{N}-\frac{\rho S V}{V_{50}+V} \\
\frac{d I}{d t} & =\frac{\beta S I}{N}+\frac{\rho S V}{V_{50}+V}-\gamma I \\
\frac{d R}{d t} & =\gamma I \\
\frac{d V}{d t} & =\kappa I-\eta V .
\end{aligned}
$$

Here $S, I$ and $R$ represent the number of individuals in each class respectively and $N$ is the total number of individuals $(N=S+I+R) . \beta$ is the person-to-person transmission parameter. Individuals can also contract the disease from the environmental reservoir with a dose related response according to a Holling type II functional (Holling, 1959) where $V_{50}$ is the infectious dose required to produce disease in $50 \%$ of those exposed and $\rho$ is the transmission parameter. Individuals recover from the disease and move into the recovered class at a rate $\gamma$ and hence $1 / \gamma$ is the average time spent in the infectious state. An infective individual sheds virus to the environmental reservoir at a rate $\kappa$ and the virus decays in the environment at a rate $\eta$. Due to the low mortality rate of hepatitis E, the relatively short time scale of interest (of the order of 6 months) and the closed nature of IDP camps, birth and death terms in the model are not required. Additionally equation (3) is not required as $S+I+R=N$ is a conserved quantity and so $R$ can be determined from $S$ and $I$.

For a disease such as hepatitis E this simple model (1)-(4) is not adequate for two reasons. Firstly, in the above model individuals are infective the moment they contract the disease. This is not suitable as hepatitis $E$ has a substantial latent period where exposed individuals are not yet infective. The addition of an exposed compartment to the model as demonstrated in Figure 1 corrects for this. The rate of progression from exposed to infective is given by $\delta$ and hence the average time spent in the exposed compartment is $1 / \delta$. Secondly, hepatitis $E$ is known to have a large proportion of people who are asymptomatic and show little or no symptoms but still shed virus and are infective (Aggarawal and Naik, 1994; Teshale et al., 2010). Since case data are only available on symptomatic cases this needs to be allowed for in the model when estimating parameters from reported cases. The infective compartment is split into symptomatic $\left(I_{s}\right)$ and asymptomatic $\left(I_{a}\right)$ individuals, shown in Figure 1. The symptomatic proportion is taken to be $\alpha$.

For hepatitis $\mathrm{E}$ the $V_{50}$ infectious dose parameter is unknown, as is the shedding rate to the environment $\kappa$. These can be combined into one unknown parameter by the change of variable $U=V / V_{50}$ which results in one scaled parameter $\omega=\kappa / V_{50}$. Under this change of variable the environmental source infection term in equations (1) and (2) which was $\rho S V /\left(V_{50}+V\right)$ now becomes $\rho S U /(1+U)$.

Additionally, it is well known that rate terms such as $-\gamma I$ in equation (2) give rise to negative exponentially distributed infectious periods (Wearing et al., 2005). This is unrealistic for a disease such as hepatitis E which has latent and infectious periods that have a more central tendency (Takahashi et al., 2007) and are better approximated by gamma distributions. To overcome this both the exposed and infectious compartments are subdivided into multiple consecutive compartments which is known to produce 


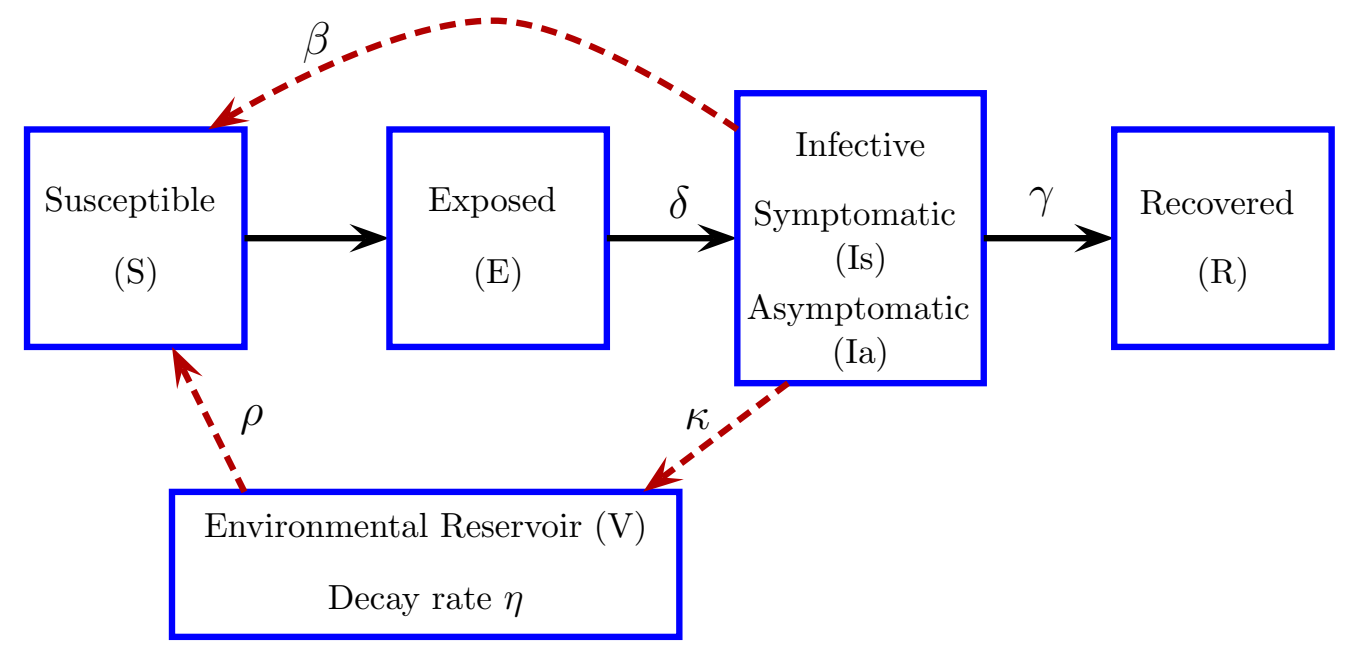

Figure 1. Schematic diagram of the model that includes person-to-person transmission, an environment reservoir, a latent (exposed) compartment and both symptomatic and asymptomatic infective compartments.

gamma distributed periods (Lloyd, 2001). The exposed compartment is divided into $m$ subgroups $\left(E_{1}\right.$, $\left.E_{2}, \ldots, E_{m}\right)$ with a rate of $m \delta$ for movement between each subgroup and similarly the infectious compartments $\left(I_{a}\right.$ and $\left.I_{s}\right)$ are subdivided in $n$ subgroups $\left(I_{a 1}, \ldots, I_{a n}\right.$ and $\left.I_{a 1}, \ldots, I_{a n}\right)$ with a rate $n \gamma$ between subgroups. This ensures the average time in each group remains $1 / \delta$ and $1 / \gamma$ respectively and that they are gamma distributed.

Taking all of the above modifications into account the governing differential equations for the model become

$$
\begin{aligned}
\frac{d S}{d t} & =-\frac{\beta S}{N} \sum_{i=1}^{n}\left(I_{s i}+I_{a i}\right)-\frac{\rho S U}{1+U} \\
\frac{d E_{1}}{d t} & =\frac{\beta S}{N} \sum_{i=1}^{n}\left(I_{s i}+I_{a i}\right)+\frac{\rho S U}{1+U}-m \delta E_{1} \\
\frac{d E_{i}}{d t} & =m \delta\left(E_{i-1}-E_{i}\right) \quad \text { for } i=2 \ldots m \\
\frac{d I_{a 1}}{d t} & =(1-\alpha) m \delta E_{m}-n \gamma I_{a 1} \\
\frac{d I_{a j}}{d t} & =n \gamma\left(I_{a j-1}-I_{a j}\right) \quad \text { for } j=2 \ldots n \\
\frac{d I_{s 1}}{d t} & =\alpha m \delta E_{m}-n \gamma I_{s 1} \\
\frac{d I_{s j}}{d t} & =n \gamma\left(I_{s j-1}-I_{s j}\right) \quad \text { for } j=2 \ldots n \\
\frac{d U}{d t} & =\omega \sum_{i=1}^{n}\left(I_{s i}+I_{a i}\right)-\eta U .
\end{aligned}
$$




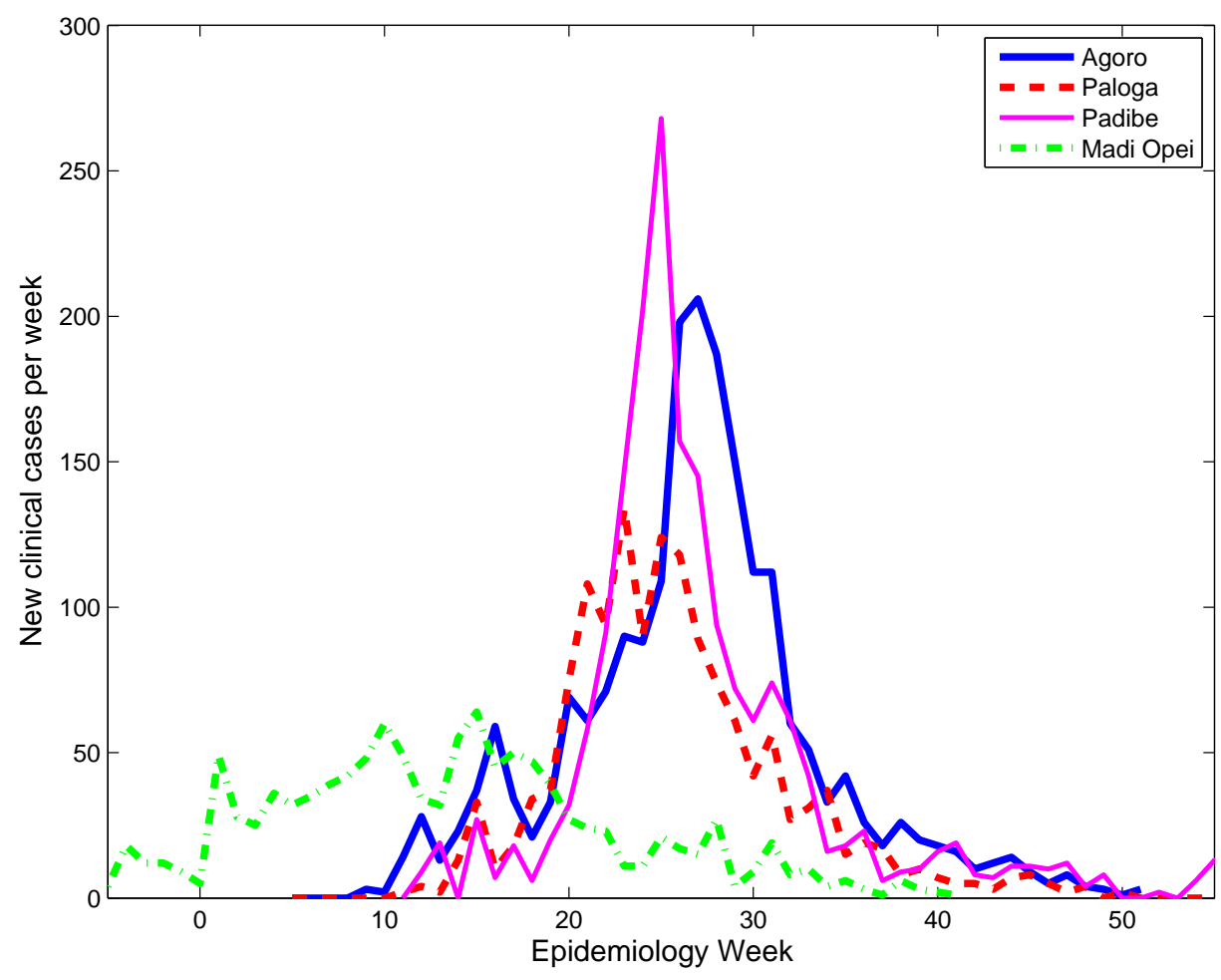

Figure 2. New clinical hepatitis E cases per week in four Ugandan IDP camps

\section{PARAMETER VALUES AND DATA}

The aetiology of hepatitis $\mathrm{E}$ is currently not well understood. What is known is that the latent period lasts on average approximately 2 weeks $(1 / \delta=2)$, and viral shedding during the infectious stage lasts on average approximately 5 weeks $(1 / \gamma=5)$ (World Health Organisation, 2001). Hepatitis $\mathrm{E}$ is thought to be more labile than hepatitis A (Balayan, 1997; Skidmore, 1999) and has a shorter persistence time in the environmental reservoir so we take the average survival time to be 4 weeks $(1 / \eta=4)$ in comparison to hepatitis A of 12 weeks (Ajelli et al., 2008). Analysis of seroprevalence survey data from the recent Ugandan outbreak (Teshale et al., 2010) indicates that the symptomatic ratio is quite low with the symptomatic proportion being approximately $\alpha=0.125$. The other parameters, $\beta, \rho$ and $\omega$ are related to the transmission dynamics and are unknown and need to be determined by fitting the model output to outbreak data.

Shown in Figure 2 are new clinical (symptomatic) cases per week in four IDP camps during the Ugandan outbreak. The three camps Agoro, Paloga and Padibe all show similar trends and timings. The fourth camp, Madi Opei, had a significantly different transmission pattern possibly due to the different timing of the outbreak. In Madi Opei the outbreak commenced earlier, during one of the wet seasons which is thought to affect the spread of hepatitis E (Balayan, 1997).

The unknown parameters are estimated using an approximate Bayesian Markov chain Monte Carlo method that has previously been applied to population models (Haario et al., 2006). In short the process to estimate a parameter $\theta$ is as follows. First a prior distribution, $\pi(\theta)$, for $\theta$ is assumed. Here we take the prior distributions to be uniform distributions over a range much larger than the expected value of the parameter. This type of uninformative prior ensures we do not bias the final posterior distribution. Next a value $\theta^{*}$ is sampled from $\pi(\theta)$, the model run with $\theta^{*}$ and the output compared to the data. The sampled $\theta^{*}$ value is then either accepted or rejected depending on how close to the data the result was, $\pi(\theta)$ is updated and the process repeated. This is undertaken using the Metropolis-Hastings algorithm 
Table 1. Average parameter values (and 95\% credible interval) from 10,000 instances of the model from the MCMC method

\begin{tabular}{|l|l|l|l|}
\hline Camp & $\rho \times 10^{2}$ & $\omega \times 10^{4}$ & $\beta / N$ \\
\hline Agoro & $9.11(9.05,9.18)$ & $7.14(5.53,9.12)$ & $0.521(0.424,0.664)$ \\
Paloga & $9.02(8.96,9.07$ & $8.29(7.78,8.77)$ & $0.488(0.462,0.514)$ \\
Padibe & $9.01(8.95,9.10)$ & $13.1(11.7,14.3)$ & $0.184(0.124,0.234)$ \\
Madi Opei & $8.73(8.70,8.79)$ & $15.2(13.8,16.5)$ & $0.011(0.004,0.035)$ \\
\hline
\end{tabular}

and full details of the method applied to systems of ordinary differential equations can be found in Haario et al. (2006). A burn-in time of 10,000 runs of the model was used to allow the parameter distributions to reach a quasi-equilibrium and the results for the parameter distributions were calculated on the next 10,000 samples of the parameters.

Mean estimates (and 95\% credible intervals) of $\rho, \omega$ and $\beta / N$ values are shown in Table 1 for the four camps and an example of the posterior distributions for the Paloga camp is shown in Figure 3. Encouragingly, consistent estimates (with narrow credible intervals) are obtained for $\rho$, the environment to individual transmission parameter, for all of the camps. The scaled shedding to the environment parameter, $\omega$, was less consistent across the camps and had a wider credible interval but were all still comparable. This variation is possibly explained by varying levels of pre-existing latrines in the camps that impacted on the shedding rate to the environment. The person-to-person parameter, $\beta$, showed the greatest variation with the Madi Opei camp being particularly different to the other camps. This is consistent with the outbreak data in Figure 2 with the Madi Opei camp having a very different distribution of cases compared to the other camps. There is some disagreement in the literature about the importance of person-toperson transmission of hepatitis E with Somani et al. (2003) (and others) stating that it is minimal whilst Teshale et al. (2010) claim it to be significant during the 2008 Ugandan outbreak although this finding has been questioned by Aggarwal (2010) and Nishiura (2010). Our results confirm this uncertainty about person-to-person transmission and this is clearly an area that needs further research to fully understand the transmission of hepatitis E in an IDP camp setting.

\section{DISCUSSION}

In internally displaced persons camps interventions are undertaken by groups such as Médecins Sans Frontières to reduce transmission of hepatitis E. These include the installation of hand washing points to reduce person-to-person transmission, water purification to reduce contraction of the disease from the environmental source and latrine building to reduce shedding of the virus by infected individuals to the environment. In terms of the parameters in the model the interventions all reduce the appropriate transmission parameter. Installation of hand washing points reduces $\beta$, water purification reduces $\rho$ and latrine construction reduces $\omega$.

Of interest is how effective these interventions are and which intervention should take precedence. In addition, interventions do not commence instantly as there are delays in their implementation. The timing of these delays are specific to each camp and depend on numerous factors including the availability of testing, thresholds for interventions to commence, hardware, manpower and other resources limitations. Once appropriate timings are decided upon, in consultation with the field epidemiologists, then the model can be run with different combinations and degrees of interventions to investigate the optimal use of resources that reduces the burden of disease in the camps.

There are two objectives when deciding on the optimal intervention strategy given the constraints around timing and resources. Firstly, the peak incidence is an important consideration as this impacts on the ability of the medical staff to cope with the peak workload. Secondly, it is important to reduce as much as possible the cumulative incidence of the disease in the camps. Investigating these aspects is the topic of current research. 
G.N. Mercer and M.R. Siddiqui, Application of a hepatitis E transmission model ...
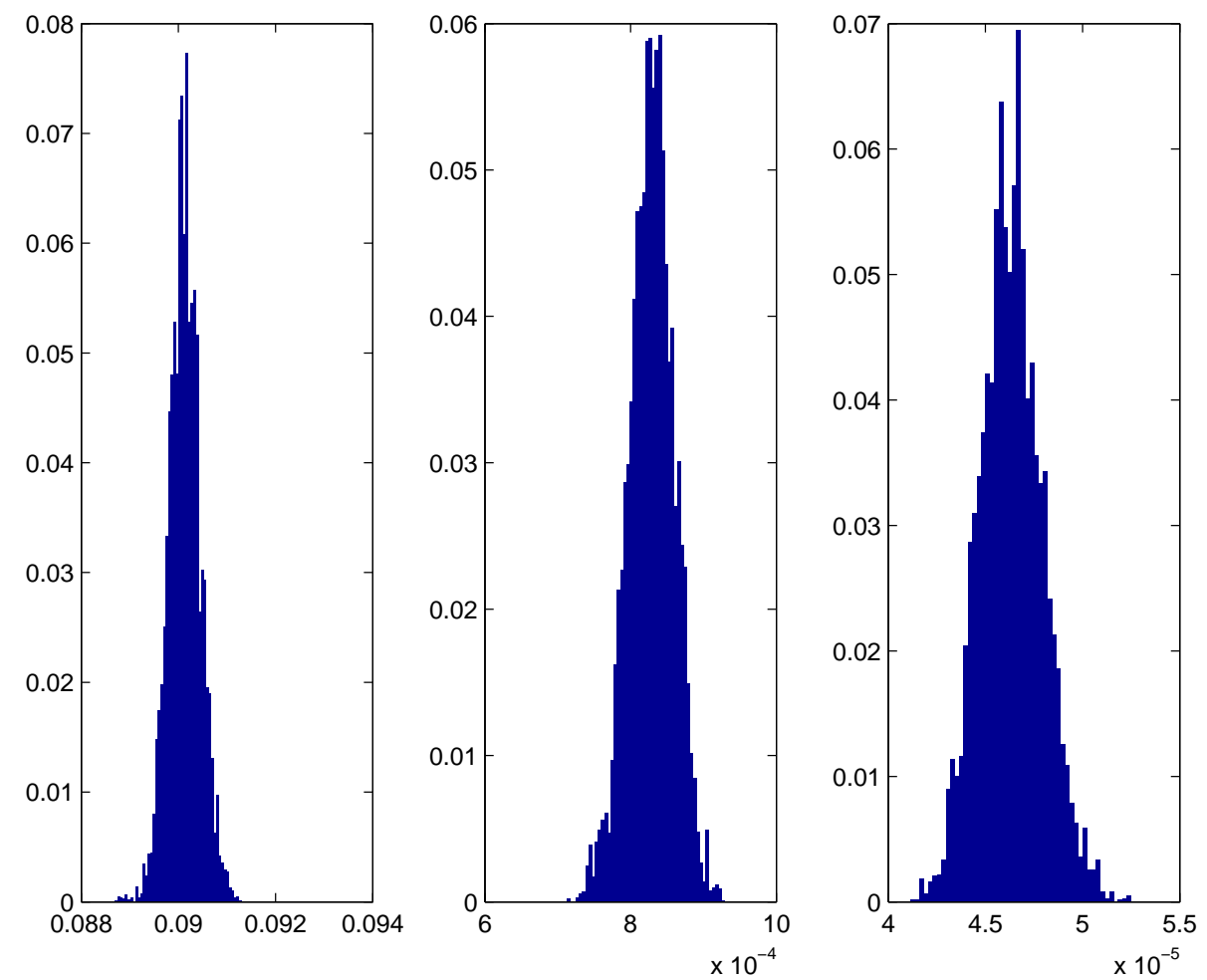

Figure 3. Posterior distributions of $\rho, \omega$ and $\beta / N$ respectively for Paloga camp data from 10,000 instances of the model

\section{REFERENCES}

Aggarawal, R. and S. R. Naik (1994). Hepatitis E: intrafamiliar transmission versus waterborne spread. Journal of Hepatology 21(5), 718-723.

Aggarwal, R. (2010). Hepatitis E virus and person-to-person transmission. Clinical Infectious Diseases $51,477-478$.

Ajelli, M., M. Iannelli, P. Manfredi, and M. L. C. degli Atti (2008, March). Basic mathematical models for the temporal dynamics of HAV in medium-endemicity Italian areas. Vaccine 26(13), 1697-707.

Andrews, A. R. and S. Basu (2011). Transmission dynamics and control of cholera in Haiti: an epidemic model. Lancet 377, 1248-1255.

Balayan, M. S. (1997, May). Epidemiology of hepatitis E virus infection. Journal of Viral Hepatitis 4(3), 155-65.

Boccia, D., J.-P. Guthmann, H. Klovstad, N. Hamid, M. Tatay, I. Ciglenecki, J.-Y. Nizou, E. Nicand, and P. J. Guerin (2006, June). High mortality associated with an outbreak of hepatitis E among displaced persons in Darfur, Sudan. Clinical Infectious Diseases 42(12), 1679-84.

Breban, R., J. M. Drake, D. E. Stallknecht, and P. Rohani (2009, 04). The role of environmental transmission in recurrent avian influenza epidemics. PLoS Comput Biol 5(4), e1000346.

Codeço, C. T. (2001, January). Endemic and epidemic dynamics of cholera: the role of the aquatic reservoir. BMC Infectious Diseases 1, 1. 
Guthmann, J.-P., H. Klovstad, D. Boccia, N. Hamid, L. Pinoges, J.-Y. Nizou, M. Tatay, F. Diaz, A. Moren, R. F. Grais, I. Ciglenecki, E. Nicand, and P. J. Guerin (2006, June). A large outbreak of hepatitis E among a displaced population in Darfur, Sudan, 2004: the role of water treatment methods. Clinical Infectious Diseases 42(12), 1685-91.

Haario, H., M. Laine, A. Mira, and E. Saksman (2006). DRAM: Efficient adaptive MCMC. Statistics and Computing 16, 339-354.

Hartley, D. M., J. G. Morris, and D. L. Smith (2006, January). Hyperinfectivity: a critical element in the ability of V. cholerae to cause epidemics? PLoS Medicine 3(1), e7.

Holling, C. S. (1959). The components of predation as revealed by a study of small-mammal predation of the european pine sawfly. Canadian Entomology 91, 293-320.

King, A. A., E. L. Ionides, M. Pascual, and M. J. Bouma (2008, August). Inapparent infections and cholera dynamics. Nature 454(7206), 877-80.

Lloyd, A. L. (2001). Destabilization of epidemic models with the inclusion of realistic distributions of infectious periods. Proc. Roy. Soc. Lond. B 268, 985-993.

Nishiura, H. (2010). Household data from the Ugandan hepatitis E virus outbreak indicate the dominance of community infection. Clinical Infectious Diseases 51, 117-118.

Rohani, P., R. Breban, D. E. Stallknecht, and J. M. Drake (2009, June). Environmental transmission of low pathogenicity avian influenza viruses and its implications for pathogen invasion. Proceedings of the National Academy of Sciences of the United States of America 106(25), 10365-9.

Siddiqui, M. (2010). MSF-OCA response to a hepatitis E outbreak in Kitgum and Pader, Uganda, 20072009: Epidemiological review. Médecins Sans Frontiéres.

Skidmore, S. J. (1999). Factors in the spread of hepatitis E. Lancet 354, 1049-1050.

Somani, R., R. Aggarwal, S. Naik, S. Srivastava, and S. Naik (2003). A serological study of intrafamiliar spread from patients with sporadic hepatitis E virus infection. Journal of Viral Hepatitis 10(6), 446449.

Takahashi, M., T. Tanaka, M. Azuma, E. Kusano, T. Aikawa, T. Shibayama, Y. Yazaki, H. Mizuo, J. Inoue, and H. Okamoto (2007). Prolonged fecal shedding of hepatitis E virus (HEV) during sporadic acute hepatitis E: Evaluation of infectivity of HEV in fecal specimens in a cell culture system. J. Clin. Microbiol. 45(11), 3671-3679.

Teshale, E. H., S. P. Grytdal, C. Howard, V. B. V, S. Kamili, J. Drobeniuc, V. R. Hill, S. Okware, D. J. $\mathrm{Hu}$, and S. Holmberg (2010). Evidence of person-to-person transmission of hepatitis E virus during a large outbreak in northern Uganda. Clinical Infectious Diseases 50, 1006-1010.

Wearing, H. J., P. Rohani, and M. J. Keeling (2005). Appropriate models for the management of infectious diseases. PLoS Medicine 2, e174.

World Health Organisation (2001). Hepatitis E. http://www.who.int/csr/disease/hepatitis. 